\title{
Tratamiento del prolapso de cúpula vaginal con sacrocolpopexia modificada utilizando dacrón de injerto vascular
}

\author{
Pío Iván Gómez S. M.D.*
}

\section{RESUMEN}

El prolapso de cúpula vaginal posthisterectomía es una entidad de difícil manejo quirúrgico; una de las técnicas más utilizadas es la fijación de la cúpula vaginal al sacro, la cual requiere hacer tunelización retroperitoneal con el riesgo de lesiones vasculares, por eso en la técnica que en este trabajo se describe se obvia la disección retroperitoneal extensa, recurriendo a un artificio realizado con el peritoneo.

MATERIALES Y METODOS: Se estudiaron 14 pacientes con prolapso de cúpula a quienes se les realizó sacrocolpopexia modificada sin tunelización retroperitoneal con dacrón de injerto vascular; se hizo seguimiento al mes, tres meses, seis meses y luego anualmente.

RESULTADOS: La edad promedio de las pacientes fue de 56.5 años, el tiempo quirúrgico promedio fue de 32.5 minutos. No hubo complicaciones intra ni postoperatorias. La estancia hospitalaria fue en promedio de 4 días. Hasta el momento no ha habido recidiva con un seguimiento entre 6 meses y 6 años, habiendo completado el 71.42\% de las pacientes un seguimiento de 4 años.

CONCLUSIONES: Esta técnica disminuye el riesgo de lesiones intraoperatorias al evitar la extensa disección retroperitoneal, sin incrementar costos, ni estancia hospitalaria, con baja posibilidad de recidiva por lo cual esta técnica puede incluirse dentro de los recursos quirúrgicos para el manejo de esta entidad.

PALABRAS CLAVES: Prolapso cúpula vaginal, sacrocolpopexia.

\section{SUMMARY}

The vaginal vault prolapse posthysterectomy is a complication with a difficult surgical treatment; the most used surgycal technique is sacropexy but it needs the aperture of the retroperitoneum with the possibility of vascular lesions. That is the reason to describe a new technique that fail on aperture of the retroperitoneum.

MATERIALS AND METHODS: 14 patients with vaginal vault prolapse were going to modificate sacral fixation, using dacron allograft. All patients underwent follow-up for one, three, six months an then each year.

RESULTS: The average age from the patients was between 44-69 years old with a mean peak over 56 years old. The average surgycal time was between 20-45 minutes $(x=32.5)$. In this study we did not have any intra o postoperative complication.

The average time of hospitalitation was 3-5 days $(x=4)$. The follow-up time was between six months to six years, with no recidives. The $71.4 \%$ of all patients was follow-up four years.

KEY WORDS: Vaginal vault prolapse, sacropexy.

\section{Introducción}

Después de una histerectomía puede aparecer el prolapso total de la cúpula vaginal o eversión masiva de la vagina, proyectándose la vagina hacia afuera como se saca al revés el dedo de un guante; esta entidad es de difícil manejo a tal punto que algunos autores la han considerado intratable (1).

La frecuencia del prolapso de la cúpula vaginal es variable, reportándose entre $2 \%$ a $18.2 \%$ (2-3).

Hay que anotar que a pesar de la alta frecuencia de esta entidad en nuestros hospitales regionales, especial* Profesor Asociado, Depto de Ginecología y Obstetricia. Univer-
sidad Nacional de Colombia. mente en zonas rurales y en los hospitales Universitarios, no se encuentra referencia de la frecuencia de esta entidad en nuestro medio.

La principal molestia de las pacientes es la incomodidad para deambular, sentarse y para la actividad sexual; cuando hay ulceraciones, pueden referir mal olor, secreción y dolor.

Se han descrito numerosas técnicas para la corrección de esta entidad, desde el uso de pesarios, pasando por la colpocleisis, hasta la suspensión de la cúpula usando materiales sintéticos o naturales, utilizando la vía vaginal o abdominal con fijación a diferentes estructuras como ligamentos de cooper, ligamentos sacrociáticos mayo- 
res, ligamento vertebral común anterior en el promontorio, disco lumbosacro y aponeurosis de rectos abdominales, entre otras (4-15).

En este trabajo se utilizó la sacrocolpopexia vía abdominal con modificaciones que evitan la tunelización retroperitoneal, disminuyendo algunos riesgos intraoperatorios y acortando el tiempo quirúrgico.

\section{Materiales y métodos}

Se estudiaron 14 pacientes que consultaron al Instituto Materno Infantil de Santafé de Bogotá y al Hospital Regional San Rafael de Facatativá y a quienes se les diagnosticó prolapso total de cúpula vaginal posthisterectomía (12 de histerectomía vaginal y 2 de histerectomía abdominal). A todas las pacientes se les realizó Sacrocolpopexia modificada, cierre del fondo de saco de Douglas y colpoperineorrafía. Se ha hecho seguimiento postoperatorio en toda las pacientes al mes, tres meses, seis meses y luego anualmente.

\section{Técnica quirúrgica (Ver figura 1)}

1. Laparotomía mediana o transversa, previa asepsiaantisepsia y colocación de un dilatador de Hegar en canal vaginal, para ascender la cúpula.

2. Apertura transversal $(2 \mathrm{cms})$ del peritoneo de la cúpula y disección hasta identificar la mucosa vaginal.

3. Apertura transversal $(2 \mathrm{cms})$ peritoneo en mitad derecha del promontorio.

4. Cierre del fondo de saco de Douglas con suturas inabsorvibles en forma de bolsa de tabaco.

5. Sutura del borde inferior de peritoneo del promontorio con borde peritoneal posterior de la cúpula.

6. Suspensión de la cúpula a ligamento vertebral común anterior en el promontorio, utilizando una tira de dacrón vascular de 1-3 cms de acuerdo con la longitud requerida.

7. Sutura de borde superior de peritoneo del promontorio con borde anterior del peritoneo de la cúpula, recubriendo el material sintético. No dejar espacios laterales que favorezcan una obstrucción intestinal; si es necesario se deben hacer cierres laterales adicionales en forma de bolsa de tabaco.

8. Cierre de laparotomía.

9. Colpoperineorrafía.

Los pasos 5 y 7 evitan la amplia disección retroperitoneal, al hacerse una peritonización por artificio.

\section{Resultados}

La edad de las pacientes estuvo entre 44 a 69 años $(\mathrm{X}=56.5)$.

El tiempo quirúrgico abdominal estuvo entre 20 y 45 minutos ( $X=32.5 \mathrm{~min})$.

En todas las pacientes se obtuvo una adecuada pexia de la vagina, dejándola en una posición fisiológica.

No hubo complicaciones intra ni postoperatorias.

La estancia Hospitalaria fue de 3-5 días $(x=4)$.

No ha habido recidivas hasta el momento, con un seguimiento entre 6 meses a 6 años, teniendo la mayoría de pacientes $(71.42 \%)$ observación de por lo menos 4 años.

\section{Discusión}

El tratamiento del prolapso total de cúpula vaginal es difícil, de ahí que se han descrito un sinnúmero de técnicas quirúrgicas; algunas tienden a fijar la vagina hacia adelante como la fijación a la fascia de los rectos abdominales o los ligamentos de cooper, lo cual da como resultado una posición antifisiológica de la vagina, con dificultades para el coito y además amplía el fondo de saco de Douglas facilitando la recidiva a expensas de un enterocele. Las técnicas vaginales que llevan un ángulo de la cúpula a los ligamentos sacroespinosos colocan la vagina en una posición fisiológica, pero además de ser una cirugía dispendiosa, requiere una vagina corta y tiende a recidivar con mayor frecuencia que las técnicas abdominales.

Dentro de las técnicas abdominales que son las que preferimos en nuestro departamento, la Sacrocolpopexia ha sido la más utilizada, por no alterar la posición de la vagina y su baja recidiva, sin embargo conlleva riesgos intraoperatorios, pues requiere realizar una tunelización retroperitoneal desde el promontorio hasta la cúpula vaginal y en esta amplia disección existe el riesgo de lesiones vasculares arteriales y venosas así como ureterales. En nuestro Departamento el Dr. Ñañez y Mercado, modificaron la técnica utilizando material de sutura para el cerclaje cervical (Cervix-set), para intentar disminuir los riesgos anteriores (16), pero en mi concepto este tipo de sutura no es el ideal pues la aguja que tiene es excesivamente grande e incluso puede facilitar lesiones de vasos hipogástricos, el material sintético utilizado debe pasarse a través del ligamento vertebral común anterior y de la mucosa vaginal, lo cual puede debilitar estas estructuras y facilitar la recidiva, de otra parte por el grosor de este poliéster trenzado los nudos son voluminosos lo cual puede coadyuvar a la reacción fibrótica postquirúrgica y ser causa de dolor.

Por lo anterior modifiqué la técnica, obviando la disección retroperitoneal y haciendo una peritonización por artificio (ver técnica), lo cual disminuye no sólo el tiempo quirúrgico, sino evita las lesiones intraoperatorias. En esta modificación se debe insistir en la necesidad de hacer un adecuado cierre del fondo de saco de Douglas, pues si se dejan espacios abiertos, al quedar la vagina a manera de "puente" intrapélvico, puede producirse una obstrucción intestinal.

En nuestra institución aprovechamos el dacrón vascular que desechan los cirujanos, reesterilizándolo a gas, por lo cual los costos no se aumentan.

Por las ventajas presentadas considero que esta técnica debe tenerse en el armamentario quirúrgico del especialista y ésta puede ser una alternativa más en el manejo de esta entidad, el cual debe siempre individualizarse de acuerdo con la paciente, la experiencia del cirujano y los recursos de la Institución.

\section{Conclusiones}

1. La sacrocolpopexia modificada disminuye el riesgo de lesiones intraoperatorias al no hacerse una extensa disección retroperitoneal. 

2. El tiempo quirúrgico se disminuye, especialmente en hospitales Universitarios.

3. Los costos no se incrementan al reutilizar el dacrón vascular sobrante de otros servicios.

4. Se disminuye la posibilidad de enterocele.
5. La vagina se fija en una posición que no limita la actividad sexual de la paciente.

6. Hasta el momento la recidiva es nula, aunque falta esperar más tiempo de observación.

\section{BIBLIOGRAFIA}

1. Weibel W. Lehrbrick der Frauenheilk Kunde, 5th Ed. Berlin, Wein. Urban u. Schwarzenberg. 1941; 38.

2. Hofmesiter FJ. Editorial Comment: prolapsed vagina. Obstet. Gynecol. 1973; 42: 773.

3. Ward GE. Ox fascia lata for reconstruction of round ligaments in correcting prolapse of the vagina. Arch. Surg. 1938; 36: 163.

4. Fletcher PF. Abdominal Colpocistopexy for complete prolapse of the vagina and bladder. Am. J. Obstet. Gynecol. 1948; 56: 41.

5. Falk HC. Uterine prolapse and prolapse of the vagina treated by sacropexy. Obstet. Gynecol. 1961; 18: 113.

6. Richter K., Albrich W. Long-term results following fixation of the vagina on the sacrospinal ligament by the vaginal route (vaginaefixatio sacrospinalis vaginalis). Am. J. Obstet. Gynecol. 1981; 141: 811.

7. Nichols DH. Transvaginal sacrospinous fixation. Pelvic. Surg. $1981 ; 1: 10$.

8. Richardson AC., Williams GO. Treatment of prolapse of the vagina following hysterectomy. Am. J. Obstet. Gynecol. 1969; 105: 90.
9. Langmade ChF. Cooper ligament repair of vaginal vault prolapse. Am. J. Obstet. Gynecol. 1965; 92: 601.

10. Ridley JH. A composite vaginal vault suspension using fascia lata. Am. J. Obstet. Gynecol. 1976; 126: 590.

11. Pelosi MP et als. Use of dermal graft in the surgycal repair of vaginal vault prolapse. Obstet. Gynecol. 1980; 55: 385.

12. O'leary JA. Ventrofixation in the management of vaginal vault prolapse. Surg. Gynecol. Obstet. 1965; 120: 1296.

13. Feldmann GB., Birnbaum SJ. Sacral colpopexy for vaginal vault prolapse. Obstet. Gynecol. 1979; 53: 399.

14. Adams HP. Total colpocleisis for pelvic eventration. Surg. Gynecol. Obstet. 1951; 92: 321

15. Calle M. Histeropexia con cinta de dacrón. Rev. Col. Obst. Ginecol. 1983; 34(5): 275-280.

16. Mercado ME. Sacrocolpopexia simplificada con Cervix-set. Memorias Curso "Complicaciones Médico-Quirúrgicas en Ginecología”. Edit. Presencia. 1989; 112-113. 